\title{
An integrated approach to the taxonomy of the genus Verticillium
}

\author{
YAN JUn, ${ }^{1} \uparrow$ PaUl D. Bridge ${ }^{1}$ and Harry C. Evans ${ }^{2 *}$ \\ ${ }^{1}$ International Mycological Institute, Ferry Lane, Kew, Surrey TW9 3AF, UK \\ ${ }^{2}$ International Institute of Biological Control, Silwood Park, Ascot, Berkshire SL5 7TA, UK
}

(Received 22 October 1990; revised 4 February 1991; accepted 13 February 1991)

\begin{abstract}
The taxonomic relationship among 64 isolates of the genus Verticillium was investigated using a range of morphological, physiological and biochemical characters with particular reference to $V$. lecanii. Forty-five isolates pertaining to the $V$. lecanii complex were compared, including isolates from arthropods (entomopathogens), rust fungi (hyperparasites) and 'saprophytic' sources. Other Verticillium species from invertebrate, fungal and plant hosts were included in the study to explore relationships within the genus. By analysing 41 characters, similarities were calculated between isolates using Gower's coefficient, and dendrograms were derived by average linkage clustering. Six cluster groups were delimited: $V$. lecanii isolates (7), mostly from homopteran insects; hyperparasitic isolates (3), including one of $V$. psalliotae, from rusts; $V$. lecanii isolates (18) mainly from insects of UK origin; $V$. lecanii isolates (7) from coffee rust; $V$. lecanii isolates (11) from scale insects, predominantly tropical; and two isolates of $V$. alboatrum. The remaining 16 isolates, mostly from Cordyceps and Torrubiella infections of tropical forest arthropods, did not fit into any cluster group and all probably represent undescribed anamorphs. A similar pattern was obtained when the electrophoretic properties of catalase isoenzymes were compared. Twelve catalase bands were identified. The taxonomic significance of these findings is discussed.
\end{abstract}

\section{Introduction}

The genus Verticillium Nees (Hyphomycetes, Deuteromycotina) is a large, heterogeneous assemblage of taxa grouped according to relatively simple and ill-defined characters. A monographic study of the genus 'is overdue but will present a task requiring a period of some years' (Gams \& Van Zaayen, 1982). Earlier, Gams (1971) redescribed the genus Verticillium and proposed the new section Prostrata to accommodate taxa without welldefined, erect conidiophores. These comprised mainly invertebrate pathogens, originally placed in the genus Cephalosporium, with teleomorphs in the Clavicipitaceae (Evans \& Samson, 1982). Balazy (1973) disagreed with these 'too far-reaching generalisations' and conserved the species with verticillate phialides in Cephalosporium. This classification has not been generally accepted (Carmichael et al., 1980) and subsequently, Gams \& Van Zaayen (1982) proposed two new sections, Nigrescentia and Albo-erecta, accommodating the plant-pathogenic

$\dagger$ Present address: Experiment Centre of Integrated Biological Control on Northern Forest Pests and Diseases of China, Huanghe St, Shenyang, China. and fungicolous species, respectively. Nevertheless, Verticillium continues to be a difficult and controversial genus (Evans \& Samson, 1986; Gams, 1988) and one of more than academic interest, since it contains parasitic species of considerable economic importance. Any name changes could have commercial repercussions, for example in the registration of new products. Verticillium lecanii (Zimm.) Viegas has long been recognized as a potential biocontrol agent of arthropod pests (Petch, 1925; Samson et al., 1988) and is currently being commercially re-evaluated (Quinlan, 1988; van der Schaaf et al., 1990). Moreover, its ability to hyperparasitize Uredinales has led to a number of studies on its possible use for the biological control of rust diseases (Spencer, 1980; Blakeman \& Fokkema, 1982; Srivastava et al., 1985; Uma \& Taylor, 1987). A wide range of isolates attributable to this species is held in the culture collection of the International Mycological Institute (IMI) and such is the variation in the species complex that eight synonymous taxa were described by the same author over a 17 year period (Gams, 1971). The main objective of the present study was to test the validity of the $V$. lecanii species concept, as defined by Gams (1971), by comparing a representative selection of IMI isolates 
using the multidisciplinary technique adopted by Bridge et al. (1989) and Mugnai et al. (1989) to resolve similar problems in the genera Penicillium and Beauveria, respectively. Biochemical and physiological, as well as morphological characters are employed in this integrated approach to distinguish between taxa. Other clavicipitaceous fungi with Verticillium-like anamorphs, isolated from tropical forest arthropods, together with several common fungicolous and plant pathogenic species, were included to test both species and generic concepts.

\section{Methods}

Isolates examined. The 64 isolates studied are listed in Table 1 under their IMI accession code and designated species name, together with details of host or substrate and geographical origin. A total of 66 isolates appears in the list but two of these were found subsequently to be duplicates $(41=25$ and $56=40)$.
Inoculation of test media. All inoculations were by means of agar plugs ( $5 \mathrm{~mm}$ diameter) containing hyphal tips from $10 \mathrm{~d}$ cultures grown on tap water agar (TWA) (Smith \& Onions, 1983).

Morphological characterization. For macromorphological studies, isolates were grown both in the dark (at $25^{\circ} \mathrm{C} \pm 2{ }^{\circ} \mathrm{C}$ ) and light (supplemented with $\mathrm{UV}$, at $30^{\circ} \mathrm{C} \pm 3{ }^{\circ} \mathrm{C}$ ) on potato dextrose agar (PDA), potato carrot agar (PCA) and malt agar (MA). Culture characteristics were assessed after 2 and 4 weeks. For micromorphological studies, isolates were grown using the slide culture technique (Booth, 1971) and examined as described previously (Mugnai et al., 1989). In addition, 3- to 5-week-old PCA and MA cultures were screened for the presence of secondary structures (chlamydospores or resting mycelium). Morphological characters were coded by the 'all present' system (Mugnai et al., 1989; Bridge \& Sackin, 1991) to account for the effect of exclusive characters.

Physiological characterization. The hydrolysis of aesculin, Tween 80, RNA and gelatin, and growth on citric and lactic acids as sole carbon sources, were assessed according to Bridge (1985). Urease activity was measured using the method of Mugnai et al. (1989). The ability to grow at reduced water activity on $\mathrm{G}_{25} \mathrm{~N}$ medium (Pitt, 1980) and at varying

Table 1. Verticillium isolates studied

\begin{tabular}{|c|c|c|c|}
\hline $\begin{array}{l}\text { Isolate no. and } \\
\text { accession code }\end{array}$ & Species designation & Host or substrate & $\begin{array}{l}\text { Country and } \\
\text { date of isolation }\end{array}$ \\
\hline $\begin{array}{l}\text { 1. IMI } 21167 \\
\text { 3. IMI } 68689 \mathrm{a} \text { ) }\end{array}$ & Verticillium lecanii (Zimm.) Viegas & Icerya purchasi (Coccidae: Homoptera) & Sri Lanka 1924 \\
\hline $\left.\begin{array}{l}\text { 4. IMI } 68689 \mathrm{~b} \\
\text { 5. IMI } 68689 \mathrm{c}\end{array}\right\} *$ & V. lecanii & Trialeurodes vaporariorum (Aleyrodidae: Homoptera) & UK 1972 \\
\hline 6. IMI 79606 & V. lecanii & Pulvinaria floccifera (Coccidae: Homoptera) & Turkey 1960 \\
\hline 7. IMI 115197 & V. lecanii & Myzus persicae (Aphididae: Homoptera) & UK 1965 \\
\hline 8. IMI 176057 & V. lecanii & Contact lens & UK 1973 \\
\hline 9. IMI 176391 & V. lecanii & Scolytus scolytus (Curculionidae: Coleoptera) & UK 1973 \\
\hline 10. IMI 179172 & V. lecanii & Macrosiphoniella sanborni (Aphididae: Homoptera) & UK 1973 \\
\hline 11. IMI 179173 & $V$. lecanii & Brachycaudus helichrysi (Aphididae: Homoptera) & UK 1973 \\
\hline 12. IMI 235048 & V. lecanii & Cecidophyopsis ribis (Acarinae: Arachnida) & UK 1979 \\
\hline 13. IMI 255033 & V. lecanii & Coccus viridis (Coccidae: Homoptera) & Sri Lanka (Lunuwila) 1981 \\
\hline 14. IMI 255034 & V. lecanii & Coccus viridis & Sri Lanka (Monergala) 1981 \\
\hline 15. IMI 268316 & V. lecanii & Thrips tabaci (Thripidae, Thysanoptera) & UK 1982 \\
\hline 16. IMI 268317 & V. lecanii & Thrips tabaci & UK 1982 \\
\hline 17. IMI 270376 & V. lecanii & Contaminated pesticide solution & UK 1982 \\
\hline 18. IMI 272211 & V. lecanii & Pteridium aquilinum (Pteridophyta) & UK 1982 \\
\hline 19. IMI 282532 & V. lecanii & Cossus cossus (Cossidae: Lepidoptera) & Italy 1984 \\
\hline 20. IMI 288986 & V. lecanii & Scale insect (Coccidae: Homoptera) & USA 1984 \\
\hline 21. IMI 293226 & V. lecanii & Laboratory solution & UK 1985 \\
\hline 22. IMI 293227 & V. lecanii & Soil & UK 1985 \\
\hline 23. IMI 293228 & V. lecanii & Leaf litter & UK 1985 \\
\hline 24. IMI 303103 & V. lecanii & Hemileia vastatrix (Coffee rust) & Colombia 1986 \\
\hline 25. IMI 304815 & $\begin{array}{l}\text { Verticillium state of Torrubiella rubra } \\
\text { Pat. \& Lagerh. }\end{array}$ & Scale insect (Coccidae: Homoptera) & Dominica 1986 \\
\hline 26. IMI 304817 & $\begin{array}{l}\text { V. lecanii state of Torrubiella } \\
\text { confragosa Mains }\end{array}$ & Scale insect (Coccidae: Homoptera) & Dominica 1986 \\
\hline 27. IMI 317424 & V. lecanii & Hemileia vastatrix & Jamaica 1987 \\
\hline 28. IMI 317425 & V. lecanii & Coffee scale (Coccidae: Homoptera) & Jamaica 1987 \\
\hline 29. IMI 317441 & V. lecanii & Coffee scale (Coccidae: Homoptera) & Jamaica 1987 \\
\hline 30. IMI 317442 & $V$. lecanii state of $T$. confragosa & Hemileia vastatrix & Jamaica 1987 \\
\hline 31. IMI 317444 & $V$. lecanii state of $T$. confragosa & Hemileia vastatrix & Jamaica 1987 \\
\hline 32. IMI 317451 & $V$. lecanii state of $T$. confragosa & Hemileia vastatrix & Jamaica 1987 \\
\hline 33. IMI 321293 & $V$. lecanii & Hemileia vastatrix & Colombia 1986 \\
\hline 35. IMI 329758 & V. lecanii & Hemileia vastatrix & Mexico 1988 \\
\hline 36. IMI 329759 & V. lecanii & Hemileia vastatrix & Mexico 1988 \\
\hline 37. IMI 331542 & $V$. lecanii state of $T$. confragosa & Penthocrates styx (Limacodidae: Lepidoptera) & Philippines 1985 \\
\hline 38. IMI 331550 & V. lecanii & Coffee scale (Coccidae: Homoptera) & Indonesia 1985 \\
\hline 39. IMI 331588 & $V$. lecanii state of $T$. confragosa & Forest scale (Coccidae: Homoptera) & Dominica 1986 \\
\hline 40. IMI 304807 & $V$. lecanii state of $T$. confragosa & Coffee scale (Coccidae: Homoptera) & Dominica 1986 \\
\hline
\end{tabular}


Table 1-continued

\begin{tabular}{|c|c|c|c|}
\hline $\begin{array}{l}\text { Isolate no. and } \\
\text { accession code }\end{array}$ & Species designation & Host or substrate & $\begin{array}{l}\text { Country and } \\
\text { date of isolation }\end{array}$ \\
\hline 41. IMI 304815 & Verticillium state of $T$. rubra & Scale insect (Coccidae: Homoptera) & Dominica 1986 \\
\hline 42. IMI 338013 & V. lecanii & Lawana conspersa (Flatidae: Homoptera) & Malaysia 1988 \\
\hline 43. IMI 338014 & V. lecanii & Scale insect (Coccidae: Homoptera) & Mexico 1988 \\
\hline 44. IMI 338015 & V. lecanii & Hemileia vastatrix & Uganda 1988 \\
\hline 45. IMI 338016 & $V$. lecanii state of $T$. confragosa & Saissetia scale (Coccidae: Homoptera) & Ecuador (Galapagos) 1976 \\
\hline 46. IMI 331547 & V. chlamydosporium Goddard & Slug eggs (Mollusca) & Brazil 1985 \\
\hline 49. IMI 317438 & V. lecanii & Scale insect (Coccidae: Homoptera) & Jamaica 1987 \\
\hline 50. IMI 317439 & V. lecanii & Citrus scale (Coccidae: Homoptera) & Jamaica 1987 \\
\hline 51. IMI 317440 & V. lecanii & Coffee scale (Coccidae: Homoptera) & Jamaica 1987 \\
\hline 53. IMI 304801 & V. lecanii & Scale insect (Coccidae: Homoptera) & Dominica 1986 \\
\hline 54. IMI 304805 & Verticillium $\mathrm{sp}$. & Bamboo scale & Dominica 1986 \\
\hline 55. IMI 304806 & V. lecanii & Citrus scale (Coccidae: Homoptera) & Dominica 1986 \\
\hline 56. IMI 304807 & $V$. lecanii state of $T$. confragosa & Coffee scale (Coccidae: Homoptera) & Dominica 1986 \\
\hline 57. IMI 304809 & V. lecanii & Homopteran & Dominica 1986 \\
\hline 58. IMI 331569 & Verticillium sp. & Armoured scale (Diaspididae: Homoptera) & Kenya 1986 \\
\hline 59. IMI 331593 & $V$. lecanii & Forest aphid (Aphididae: Homoptera) & Ecuador 1976 \\
\hline 61. IMI 331563 & V. hemipterigenum Petch & Bamboo scale (Coccidae: Homoptera) & Trinidad 1985 \\
\hline 62. IMI 62464 & V. alboatrum Reinke \& Berthold & Humulus lupulus (Hops, Cannabidaceae) & UK 1955 \\
\hline 63. IMI 172738 & V. alboatrum & Lycopersicon esculentum (Tomato, Solanaceae) & UK 1971 \\
\hline 64. IMI 90246 & $V$. psalliotae Treschow & Unidentified rust & India 1961 \\
\hline 65. IMI 331549 & Verticillium sp. state of Torrubiella sp. & Moth pupa (Lepidoptera) & Indonesia 1985 \\
\hline 67. IMI 338017 & Verticillium sp. state of Cordyceps $\mathrm{sp}$. & Beetle larva (Coleoptera) & Ecuador 1974 \\
\hline 68. IMI 331576 & Verticillium sp. state of Cordyceps sp. & Bagworm larva (Psychidae: Lepidoptera) & Ecuador 1975 \\
\hline 69. IMI 338018 & $\begin{array}{l}\text { Verticillium sp. state of Cordyceps } \\
\text { calocerioides Berk. \& Curt. }\end{array}$ & Spider (Araneida: Arachnida) & Brazil 1978 \\
\hline 70. IMI 331582 & Verticillium sp. state of Cordyceps sp. & Beetle larva (Elateridae: Coleoptera) & Brazil 1978 \\
\hline 71. IMI 331591 & Verticillium $\mathrm{sp}$. & Spider (Araneida: Arachnida) & Ecuador 1974 \\
\hline 72. IMI 338019 & Verticillium sp. state of Cordyceps & Spider (Araneida: Arachnida) & Brazil 1979 \\
\hline 73. IMI 331580 & Verticillium sp. state of Cordyceps sp. & Bagworm larva (Psychidae: Lepidoptera) & Brazil 1978 \\
\hline
\end{tabular}

* Isolates separated on basis of secondary metabolites produced.

temperatures $\left(15,20,25,30\right.$ and $\left.37^{\circ} \mathrm{C}\right)$ on MA (Smith \& Onions, 1983) was also tested. Growth with sodium nitrite was assessed on nitrite/ sucrose agar (Frisvad, 1981). Growth and pigmentation on media containing ammonium oxalate and mannitol were evaluated as previously described (Bridge et al., 1986; Brayford \& Bridge, 1989). The ability to grow in the presence of crystal violet $\left(0.04\right.$ and $\left.0.2 \mathrm{~g} \mathrm{l}^{-1}\right)$ and $\mathrm{CuSO}_{4}\left(0.5 \mathrm{~g} \mathrm{l}^{-1}\right)$ was tested on Czapek agar $(\mathrm{CZ})$. The inhibitors were added to the autoclaved medium through a sterilization filter $(0.45 \mu \mathrm{m}$ pore size). Production of the extracellular enzymes $\beta$-glucosidase, $\beta$-galactosidase, $N$-acetyl- $\beta$-D-glucosaminidase and chitobiosidase was determined in culture fluid from 9-d-old cultures grown on glucose-yeast extract medium (GYM) (Mugnai et al., 1989), with 4methylumbelliferone-substituted substrates (Barth \& Bridge, 1989).

Catalase isoenzymes were detected by electrophoresis from cell-free extracts of 55 representative isolates, prepared as described by Mugnai et al. (1989). Electrophoresis was carried out on samples containing $50 \mu \mathrm{g}$ protein in $1 \mathrm{~mm}$ thick, $80 \times 90 \mathrm{~mm}$, vertical, $8.2 \%(\mathrm{w} / \mathrm{v})$ acrylamide gels with a Tris-citrate/Tris-glycine buffer system (Mugnai et al., 1989). Catalase activity was visualized by soaking gels in hydrogen peroxide and staining in a ferric chloride/potassium ferricyanide mixture (Woodbury et al., 1971).

Data analysis. All characterization tests were performed in duplicate; further tests were undertaken in the case of discrepant results. A basic three-point coding system of 0 for negative, 2 for positive and 1 for weak results (see footnote, Table 2) was used for most characters. Tween 80 hydrolysis results, recorded at 7 and $14 \mathrm{~d}$, growth and pigmentation on nitrite agar, and the two concentrations of crystal violet were each combined to give five-point quantitative scales. Similarities between isolates were calculated using Gower's coefficient, negative cross-matches being excluded, and dendrograms were derived by unweighted pair group method analysis (UPGMA), average linkage-clustering (Sneath \& Sokal, 1973). All computations were carried out with the MINIPAC numerical taxonomy package on an Amstrad PCW8512 microcomputer (Mugnai et al., 1989).

\section{Results}

The clusters obtained by analysing all the morphological and physiological data (41 characters) in dendrogram form are shown as groups in Tables 2 and 3 . All isolates were able to hydrolyse aesculin and Tween 80 , and were able to grow on mannitol, lactic and citric acids; none grew at $37^{\circ} \mathrm{C}$. These characters were therefore excluded from the analysis. Similarly, growth data at $20^{\circ} \mathrm{C}$ and $25^{\circ} \mathrm{C}$ showed no significant differences and were not included.

Six cluster groups can be delimited from the dendrogram (Fig. 1) based on 21 morphological and 20 physiological characters (Table 2): group I, V. lecanii isolates (7) from insects, mostly Aphididae; group II, rust hyperparasites (3), including one isolate of $V$.psalliotae; group III, isolates (18) of $V$. lecanii mainly from insects and mainly from the UK; group IV, $V$. lecanii isolates (7) 
Table 2. Numbers of isolates positive for each attribute in cluster groups

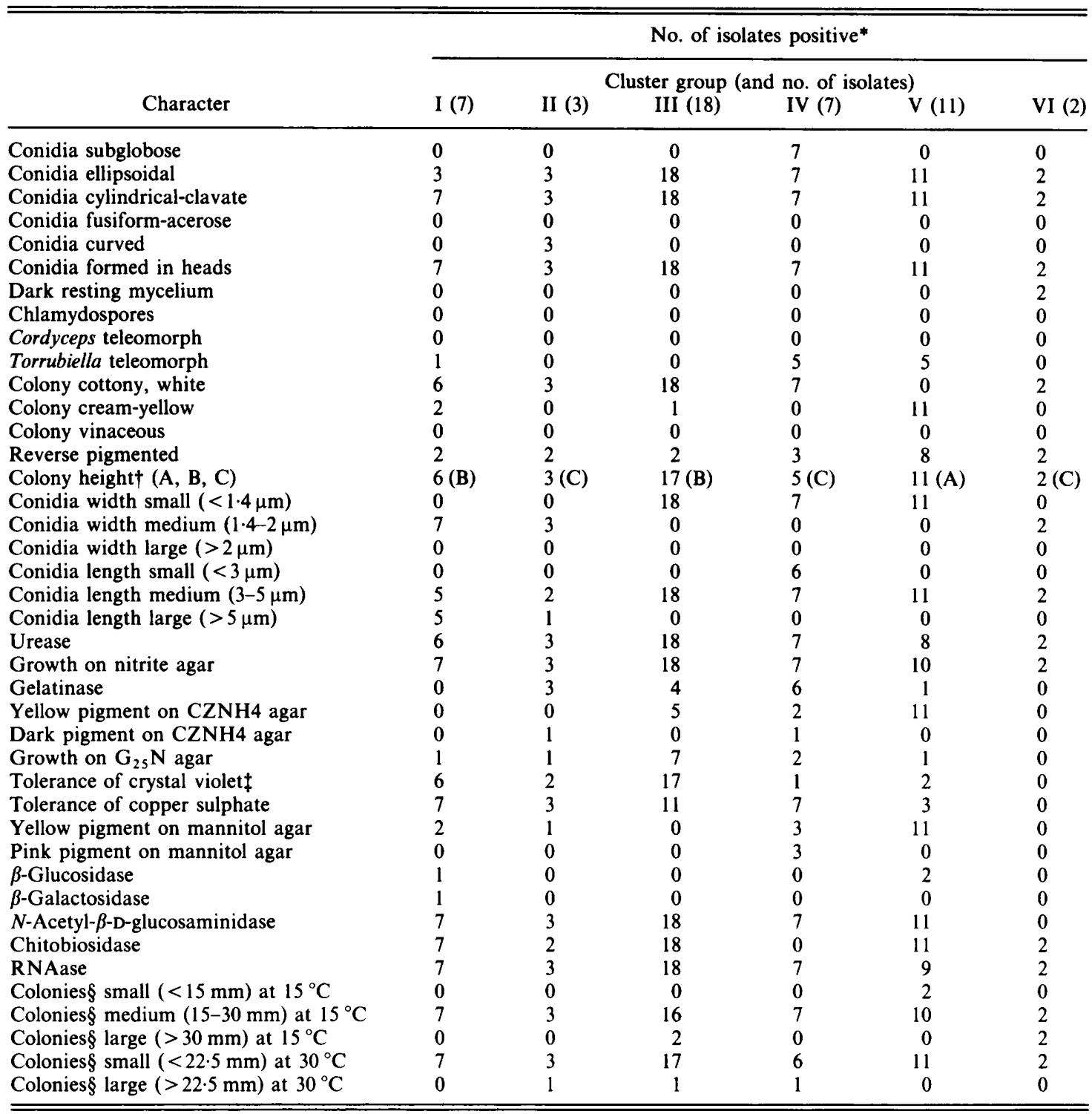

* Results of 1 (weakly positive) included as positive results.

† Colony height graded according to dominant type: A, low, appressed; B, not appressed, weakly floccose; C, strongly floccose.

$\ddagger$ Five-point scale based on both concentrations (see Methods)

$\S$ On malt agar.

from coffee rust; group $\mathrm{V}, \mathrm{V}$. lecanii isolates (11) from insects, predominantly tropical scale insects; and group VI, two isolates of $V$. alboatrum. The plant-pathogenic isolates of group VI can be separated from other isolates by the absence of $N$-acetyl- $\beta$-D-glucosaminidase, $\beta$-glucosidase, $\beta$-galactosidase and gelatinase activity. The failure of group VI isolates to grow on $\mathrm{G}_{25} \mathrm{~N}$, and to tolerate crystal violet and $\mathrm{CuSO}_{4}$ also indicates that $V$. alboatrum is physiologically distinct from the other Verticillium species examined. Morphologically, this species can readily be distinguished from the other isolates on the basis of the dark resting mycelium produced in culture. Mycelial form and colour also distinguish $V$. lecanii isolates of group $\mathrm{V}$ (ex tropical Coccidae) from those of groups III (ex mainly European insects) and IV (ex coffee rust): colonies of groups III and IV isolates are white and cottony; colonies of group V isolates are citron yellow, compact and appressed. Significantly, none of the isolates from coffee rust had chitobiosidase activity in contrast to the majority of 
Table 3. Characteristics of ungrouped isolates

\begin{tabular}{|c|c|c|c|c|c|c|c|c|c|c|c|c|c|c|c|c|}
\hline \multirow[b]{2}{*}{ Character } & \multicolumn{16}{|c|}{ Isolate no. } \\
\hline & 54 & 14 & 72 & 45 & 46 & 42 & 67 & 71 & 25 & 61 & 65 & 68 & 73 & 69 & 70 & 58 \\
\hline Conidia subglobose & - & + & - & - & + & - & + & - & - & - & - & - & - & - & + & - \\
\hline Conidia ellipsoidal & + & + & - & + & + & + & - & + & - & - & - & - & - & - & - & + \\
\hline Conidia cylindrical-clavate & + & + & + & + & - & + & - & - & + & - & + & + & + & + & + & + \\
\hline Conidia fusiform-acerose & + & - & - & - & - & - & - & - & - & - & + & - & - & - & - & + \\
\hline Conidia curved & + & - & - & - & - & - & - & - & + & + & + & - & - & - & - & + \\
\hline Conidia formed in heads & + & + & - & + & + & + & - & + & + & - & + & - & - & - & + & + \\
\hline Dark resting mycelium & - & - & - & - & - & - & - & - & - & - & - & - & - & - & - & - \\
\hline Chlamydospores & - & - & - & - & + & - & + & - & - & - & - & - & - & - & - & - \\
\hline Cordyceps teleomorph & - & - & + & - & + & - & + & + & - & - & - & + & + & + & + & - \\
\hline Torrubiella teleomorph & - & - & - & - & - & - & - & - & + & + & + & - & - & - & - & + \\
\hline Colony cottony, white & + & + & + & + & + & + & + & + & - & + & + & + & + & - & + & - \\
\hline Colony cream-yellow & - & - & - & - & - & - & - & - & - & - & - & - & - & - & - & + \\
\hline Colony vinaceous & - & - & - & - & - & - & - & - & + & - & - & + & + & - & - & - \\
\hline Reverse pigmented & - & - & - & - & + & - & - & - & + & - & - & + & + & + & + & + \\
\hline Colony height $\dagger(\mathrm{A}, \mathrm{B}, \mathrm{C})$ & B & B & B & $\mathrm{C}$ & $\mathrm{C}$ & B & B & B & A & B & $\mathrm{C}$ & $\mathrm{C}$ & $\mathrm{C}$ & $\mathrm{C}$ & A & A \\
\hline Conidia width small $(<1.4 \mu \mathrm{m})$ & + & + & + & + & + & + & - & - & - & - & + & - & - & + & - & $t$ \\
\hline Conidia width medium $(1.4-2 \mu \mathrm{m})$ & - & - & - & - & - & - & + & + & + & + & - & + & + & - & - & - \\
\hline Conidia width large $(>2 \mu \mathrm{m})$ & - & - & - & - & - & - & - & - & - & - & - & - & - & - & + & - \\
\hline Conidia length small $(<3 \mu \mathrm{m})$ & - & + & - & - & + & - & + & + & - & - & - & - & - & - & + & - \\
\hline Conidia length medium $(3-5 \mu \mathrm{m})$ & + & + & + & - & + & + & - & - & - & - & - & - & - & - & - & + \\
\hline Conidia length large $(>5 \mu \mathrm{m})$ & - & - & - & + & - & - & - & - & + & + & + & + & + & + & - & - \\
\hline Urease & - & + & + & - & + & + & + & + & + & + & + & - & - & + & + & - \\
\hline Growth on nitrite agar & + & + & + & + & + & + & + & + & + & + & + & + & + & + & + & + \\
\hline Gelatinase & - & + & + & - & - & + & - & + & - & - & - & - & - & - & - & - \\
\hline Yellow pigment $\mathrm{CZNH} 4$ agar & - & - & + & - & - & - & - & - & - & - & - & - & - & + & - & + \\
\hline Dark pigment on $\mathrm{CZNH} 4$ agar & - & - & - & - & - & - & + & - & + & + & + & + & + & - & - & - \\
\hline Growth on $\mathrm{G}_{25} \mathrm{~N}$ agar & - & - & - & - & - & - & - & - & - & - & - & - & - & - & - & + \\
\hline Tolerance of crystal violet & - & - & + & + & - & - & + & + & - & - & - & + & + & - & - & + \\
\hline Tolerance of copper sulphate & + & + & + & + & + & - & + & + & - & - & - & - & - & - & - & - \\
\hline Yellow pigment on mannitol agar & - & - & - & - & - & + & - & - & - & - & - & - & - & - & - & + \\
\hline Pink pigment on mannitol agar & - & - & - & + & + & - & + & - & + & - & + & + & + & - & - & - \\
\hline$\beta$-Glucosidase & - & - & - & - & - & - & - & - & - & - & + & - & - & - & - & - \\
\hline$\beta$-Galactosidase & - & - & - & - & - & + & - & - & - & - & - & - & - & - & - & + \\
\hline$N$-Acetyl- $\beta$-D-glucosaminidase & + & + & + & + & + & + & + & + & + & + & - & - & - & + & + & + \\
\hline Chitobiosidase & + & + & + & + & - & - & - & + & + & - & - & + & + & + & + & - \\
\hline RNAase & + & + & + & + & + & + & + & + & + & + & + & + & + & + & + & + \\
\hline Colonies§ small $(<15 \mathrm{~mm})$ at $15^{\circ} \mathrm{C}$ & - & - & - & - & - & - & - & - & - & + & - & + & + & - & + & - \\
\hline Colonies§ medium $(15-30 \mathrm{~mm})$ at $15{ }^{\circ} \mathrm{C}$ & + & - & - & + & + & + & + & + & + & - & + & - & - & + & - & + \\
\hline Colonies $\S$ large $(>30 \mathrm{~mm})$ at $15^{\circ} \mathrm{C}$ & - & + & - & - & - & - & - & - & - & - & - & - & - & - & - & - \\
\hline Colonies§ small $(<22.5 \mathrm{~mm})$ at $30^{\circ} \mathrm{C}$ & + & + & + & + & - & - & + & - & + & + & + & + & + & + & + & + \\
\hline Colonies $\S$ large $(>22.5 \mathrm{~mm})$ at $30^{\circ} \mathrm{C}$ & - & - & - & - & + & + & - & + & - & - & - & - & - & - & - & - \\
\hline
\end{tabular}

Symbols refer to footnotes in Table 2.

isolates from insects (groups I, III, V). Sixteen of the isolates could not be grouped and their combinations of characters are shown in Table 3. Most of these isolates are undescribed but three isolates received as $V$. lecanii $(14,42,45)$ are included in this category. A similar grouping pattern was obtained when catalase activity was analysed and twelve different bands were distinguished (Table 4). The electrophoretic banding patterns are represented schematically in Fig. 2 and there is a consistent distinction between $V$. lecanii isolates from insects and those from coffee rust, with the exception of isolate 33 from coffee rust in Colombia. This may be explained if isolate 33 originated from an endemic insect host and had adapted to the rust following its recent arrival in the country (Fernandez et al., 1986). Similarly, the two $V$. alboatrum isolates $(62,63)$ were distinctive in banding pattern, having a single band not shared by any other isolate. The ungrouped isolates listed in Table 3 can also be separated on the basis of their catalase activity. There are, however, some anomalous results, for example several isolates designated as $V$. lecanii $(14,42,45)$ which do not fit into any of the relevant groups, as shown in the analysis of morphological and physiological characters. In addition, two other $V$. lecanii isolates $(35$, 44) fall within the $V$. psalliotae group and recent SEM studies of conidiogenesis have confirmed that they do not belong in the $V$. lecanii complex (H. C. Evans \& Y. Jun, unpublished results). 


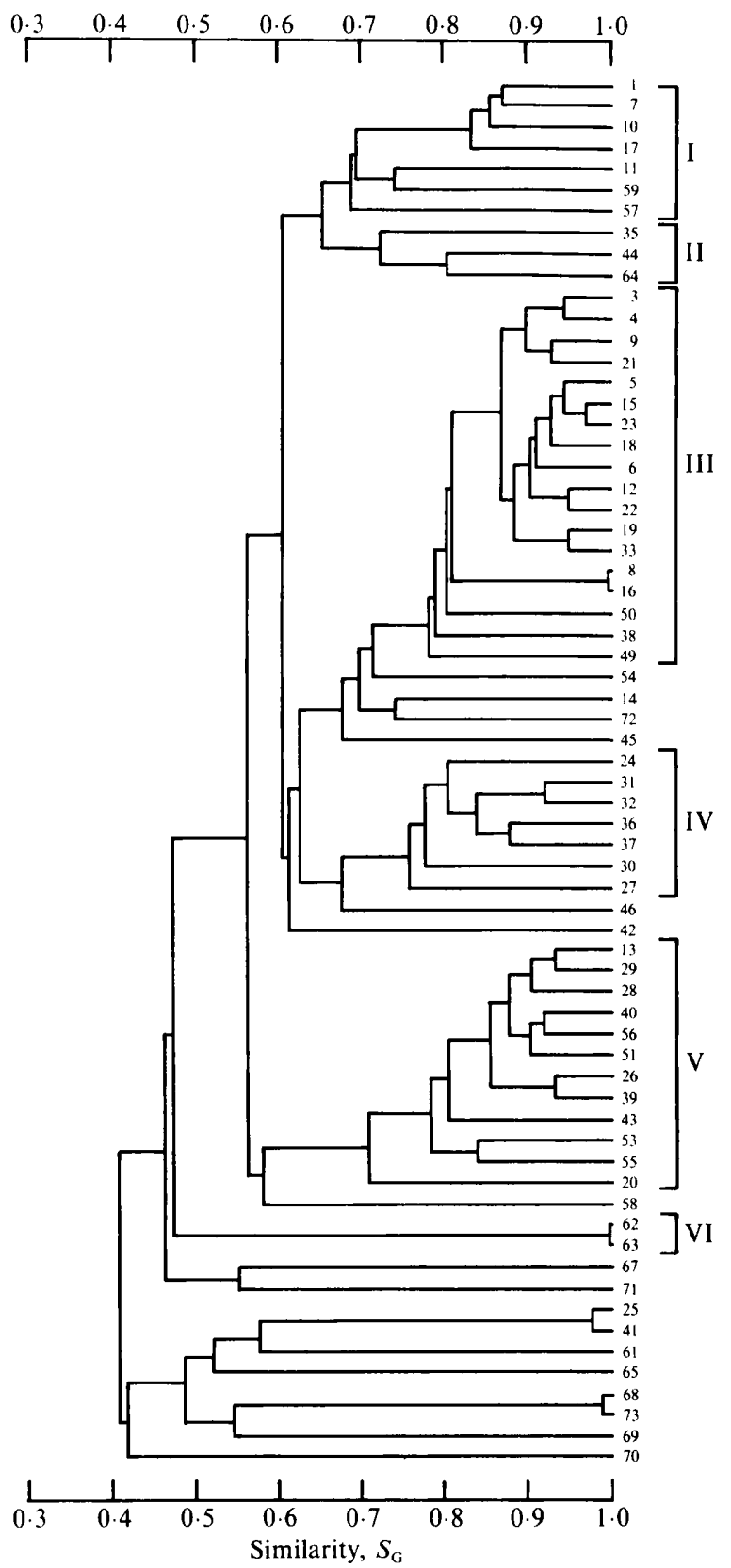

Fig. 1. UPGMA dendrogram based on 41 morphological and physiological characters. Similarities derived from Gower's coefficient discounting matching negative results, showing grouped and ungrouped isolates.
Table 4. Numbers of isolates giving catalase isoenzyme bands

\begin{tabular}{|c|c|c|c|c|c|c|c|c|c|c|c|c|c|}
\hline \multirow{2}{*}{$\begin{array}{l}\text { Cluster } \\
\text { group* }\end{array}$} & \multirow{2}{*}{$\begin{array}{l}\text { Isolates } \\
\text { included }\end{array}$} & \multicolumn{12}{|c|}{ Catalase bands } \\
\hline & & 1 & 2 & 3 & 4 & 5 & 6 & 7 & 8 & 9 & 10 & 11 & 12 \\
\hline I & $\underset{59}{1,7,10,17,57}$ & 2 & 0 & 0 & 0 & 6 & 0 & 0 & 0 & 0 & 6 & 0 & 0 \\
\hline II & $35,44,64$ & 3 & 0 & 0 & 0 & 3 & 0 & 0 & 0 & 3 & 0 & 0 & 0 \\
\hline III & $\begin{array}{c}3,4,5,6,12,15, \\
16,18,19,22, \\
23,33,38\end{array}$ & 7 & 0 & 0 & 0 & 13 & 0 & 0 & 0 & 0 & 13 & 0 & 0 \\
\hline IV & $\begin{array}{c}24,27,30,31 \\
32,36,37\end{array}$ & 0 & 0 & 0 & 0 & 6 & 0 & 0 & 0 & 0 & 0 & 7 & 0 \\
\hline V & $\begin{array}{l}13,20,26,28 \\
29,33,51,53 \\
55\end{array}$ & 3 & 0 & 0 & 0 & 9 & 0 & 0 & 0 & 0 & 9 & 0 & 0 \\
\hline VI & 62,63 & 0 & 0 & 0 & 0 & 0 & 0 & 0 & 0 & 0 & 0 & 0 & 2 \\
\hline $\begin{array}{l}\text { Ungrouped } \\
\text { isolates }\end{array}$ & $\begin{array}{l}58 \\
54 \\
14 \\
72 \\
45 \\
46 \\
42 \\
67 \\
71 \\
25 \\
61 \\
65 \\
68 \\
69 \\
70\end{array}$ & $\begin{array}{l}0 \\
0 \\
0 \\
0 \\
0 \\
0 \\
0 \\
0 \\
0 \\
1 \\
0 \\
0 \\
0 \\
1 \\
1\end{array}$ & $\begin{array}{l}0 \\
1 \\
0 \\
0 \\
0 \\
0 \\
0 \\
0 \\
0 \\
0 \\
0 \\
0 \\
1 \\
0 \\
0\end{array}$ & $\begin{array}{l}0 \\
0 \\
0 \\
0 \\
0 \\
0 \\
0 \\
0 \\
0 \\
1 \\
1 \\
0 \\
0 \\
0 \\
0\end{array}$ & $\begin{array}{l}0 \\
0 \\
0 \\
0 \\
0 \\
0 \\
0 \\
0 \\
0 \\
0 \\
0 \\
0 \\
1 \\
0 \\
0\end{array}$ & $\begin{array}{l}0 \\
0 \\
0 \\
0 \\
1 \\
0 \\
0 \\
0 \\
0 \\
0 \\
0 \\
1 \\
0 \\
0 \\
0\end{array}$ & $\begin{array}{l}0 \\
0 \\
0 \\
0 \\
0\end{array}$ & $\begin{array}{l}0 \\
0 \\
0 \\
0\end{array}$ & $\begin{array}{l}1 \\
0 \\
0 \\
1 \\
0 \\
1 \\
0 \\
1 \\
0 \\
1 \\
1 \\
1 \\
0 \\
1 \\
1\end{array}$ & $\begin{array}{l}0 \\
1 \\
0 \\
0 \\
0 \\
0 \\
0 \\
0 \\
0 \\
0 \\
0 \\
0 \\
0 \\
0 \\
0\end{array}$ & $\begin{array}{l}0 \\
0 \\
0 \\
0 \\
1 \\
0 \\
0 \\
0 \\
0 \\
0 \\
0 \\
0 \\
0 \\
0 \\
0\end{array}$ & $\begin{array}{l}0 \\
0 \\
1 \\
0 \\
0 \\
0 \\
0 \\
0 \\
0 \\
0 \\
0 \\
0 \\
0 \\
0 \\
0\end{array}$ & $\begin{array}{l}0 \\
0 \\
0 \\
0 \\
0 \\
0 \\
0 \\
0 \\
0 \\
0 \\
0 \\
0 \\
0 \\
0 \\
0\end{array}$ \\
\hline
\end{tabular}

- Cluster groups as designated in Fig. 1.

\section{Discussion}

The results of the present study show that there are highly significant physiological as well as morphological differences between $V$. alboatrum and Verticillium species pertaining to the section Prostrata, which includes both entomopathogenic and fungicolous species. There is evidence that $V$. alboatrum may behave as a mycoparasite (Barron \& Fletcher, 1970), having the ability to penetrate and grow within the conidiophores of other fungi. However, the type of mycoparasitism ascribed to $V$. alboatrum appears to differ from the lytic mechanism

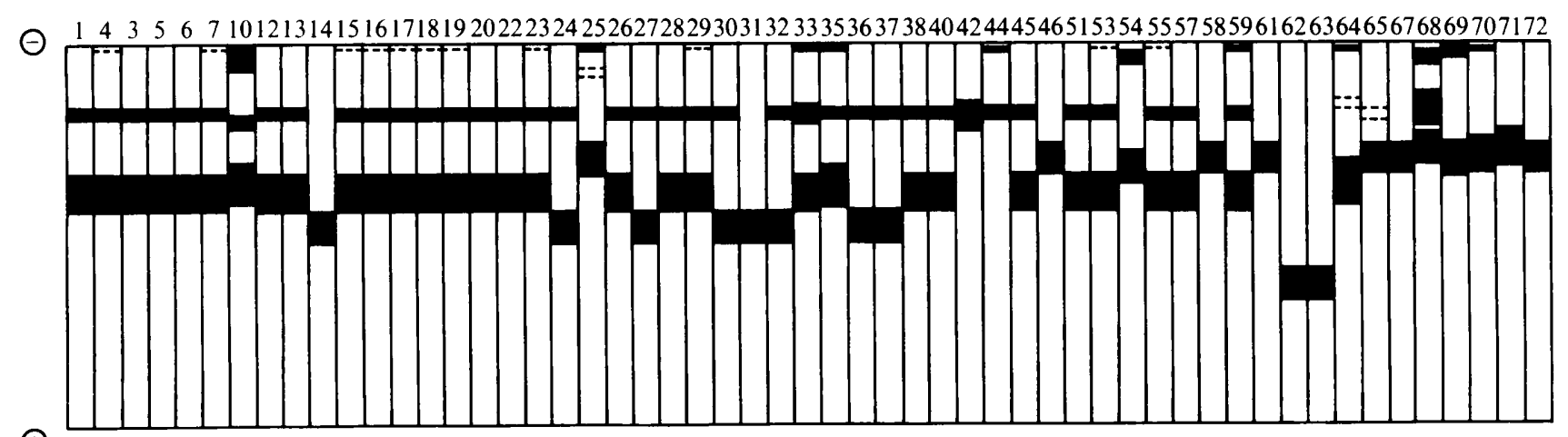

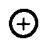

Fig. 2. Schematic representation of electrophoretic data based on catalase activity of 55 representative strains of Verticillium. 
exhibited by strains of $V$. lecanii hyperparasitic on rusts (Garcia Acha et al., 1965). Moreover, fungal-lysing enzymes were absent from the plant-pathogenic Verticillium species tested, including $V$. alboatrum. The data presented here demonstrate clear differences in enzyme composition and patterns between taxa of the section Prostrata and $V$. alboatrum.

Isolates of groups I, III, IV and V all fall within the morphological concept of the $V$. lecanii complex. However, there are sufficient physiological and biochemical characters included in the study to demonstrate clear distinctions within this complex which correlate with host and geographical origin. Similar results were obtained when the physiological and biochemical characters of a range of isolates of Beauveria bassiana (Bals.) Vuill. were subjected to principal components analysis (Mugnai et al., 1989). B. bassiana isolates from lepidopteran hosts, and to a lesser extent from hymenopteran hosts, clustered together whilst isolates on other hosts from the same collecting locality, as well as from other geographical regions, showed no grouping. The $V$. lecanii isolates from Coccidae (group V) are morphologically distinct, having short or appressed, yellow mycelia and compact phialides, whilst isolates belonging to groups $\mathbf{I}$, III and IV produce abundant white cotton-like, aerial mycelium. The group II hyperparasites are also readily distinguished from the group IV coffee rust hyperparasites on enzyme characters, and these isolates are closer to $V$. psalliotae. This species has previously been reported as a mycoparasite of coffee rust ( Lim \& Nik, 1983) and the association of two Verticillium species with this rust has undoubtedly led to misidentifications in the past, as in the case of isolates 35 and 44 . Whether or not these $V$. lecanii groups should be assigned separate taxonomic status, at the varietal or sub-species level, is open to debate but the grouping shown here clearly demonstrates the existence of subgroups within nomen species $V$. lecanii and probably explains why so many synonyms have appeared in the literature (Gams, 1971; Balazy, 1973; Brady, 1979).

The miscellaneous Verticillium isolates, including some designated as $V$. lecanii, predominantly from tropical arthropod hosts, proved to be ungrouped and all may represent separate taxa, the majority of which remain to be described (H. C. Evans \& Y. Jun, unpublished results).

Isolates 25,41 and $68,73 \mathrm{do}$, in fact, cluster together (see Fig. 1), but the former pair are duplicates of the same isolate and the latter pair represent different geographical isolates from the same lepidopteran host. These results confirm the validity of the integrated or multidisciplinary approach adopted here and provide a reliable and reproducible method for identification of fungal taxa. Whilst Gams (1988) recognized that it would be 'highly desirable' to split the genus Verticillium, more physiological and biochemical characters, in particular enzyme patterns and DNA composition, will be required to support the case for generic separation of the entomopathogenic, fungicolous and plant-pathogenic sections.

The senior author would like to thank the Gesellschaft für Technische Zusammenarbeit (GTZ, Germany) for the provision of a training scholarship, and the Director of the International Mycological Institute for laboratory facilities.

\section{References}

BALAZY, S. (1973). A review of entomopathogenic species of the genus Cephalosporium Corda (Mycota, Hyphomycetales). Bulletin de la Société des Amis des Sciences et des Lettres de Poznan 14, 101-137.

BARRON, G. L. \& FleTCHER, J. T. (1970). Verticillium albo-atrum and V. dahliae as mycoparasites. Canadian Journal of Botany 48, 1137-1139.

BARTH, M. G. M. \& BRIDGe, P. D. (1989). 4-Methylumbelliferyl substituted compounds as fluorogenic substrates for fungal extracellular enzymes. Letters in Applied Microbiology 9, 177-179.

Blakeman, J. P. \& Fokkema, N. J. (1982). Potential for biological control of plant diseases on the phylloplane. Annual Review of Phytopathology 20, 167-192.

BOOTH, C. (1971). Introduction to general methods. Methods in Microbiology 4, 1-47.

Brady, B. H. K. (1979). Verticillium lecanii. CMI Descriptions of Pathogenic Fungi and Bacteria no. 610. Kew: Commonwealth Mycological Institute.

BRAYFORD, D. \& BRIDGE, P. D. (1989). Differentiation of Fusarium oxysporum from Fusarium solani by growth and pigmentation on media containing sugar alcohols. Letters in Applied Microbiology 9 , 9-12.

BRIDGE, P. D. (1985). An evaluation of some physiological and biochemical methods as an aid to the characterization of species of Penicillium subsection Fasciculata. Journal of General Microbiology 131, 1887-1895.

Bridge, P. D. \& SACKin, M. J. (1991). Stability of classification of filamentous fungi under changes in character coding strategy. Mycopathologia (in the Press).

Bridge, P. D., Hawksworth, D. L., Kozakiewicz, Z., Onions, A. H. S. \& Paterson, R. R. M. (1986). Morphological and biochemical variation in single isolates of Penicillium. Transactions of the British Mycological Society 87, 389-396.

Bridge, P. D., Hawksworth, D. L., Kozakiewicz, Z., Onions, A. H. S., Paterson, R. R. M., Sackin, M. J. \& Sneath, P. H. A. (1989). A reappraisal of the terverticillate penicillia using biochemical, physiological and morphological features. I. Numerical taxonomy. Journal of General Microbiology 135, 2941-2966.

Carmichael, J. W., Kendrick, W. B., Conners, I. L. \& Singer, L. (1980). Genera of Hyphomycetes. Edmonton: University of Alberta Press.

Evans, H. C. \& Samson, R. A. (1982). Entomogenous fungi from the Galapagos Islands. Canadian Journal of Botany 60, 2325-2333.

Evans, H. C. \& Samson, R. A. (1986). The genus Verticillium: taxonomic problems in species with invertebrate hosts. In Fundamental and Applied Aspects of Invertebrate Pathology, pp. 186-189. Edited by R. A. Samson, J. M. Vlak and D. Peters. Wageningen, Netherlands: Foundation of the Fourth International Colloquium of Invertebrate Pathology.

Fernandez, B., Waller, J. M. \& Cookman, G. P. (1986). Coffee leaf rust (Hemileia vastatrix Berk. and $\mathrm{Br}$.) in Colombia. In Proceedings of the British Crop Protection Conference 1986, pp. 377-383. Thornton Heath, Surrey: British Crop Protection Council Publications.

Frisvad, J. C. (1981). Physiological criteria and mycotoxin production as aids in identification of common asymmetric penicillia. Applied and Environmental Microbiology 41, 568-579. 
GAMS, W. (1971). Cephalosporium-artige Schimmelpilze (Hyphomycetes). Stuttgart: G. Fischer.

GaMs, W. (1988). A contribution to the knowledge of nematophagous species of Verticillium. Netherlands Journal of Plant Pathology 94, 123-148.

Gams, W. \& VAN ZAAYEN, A. (1982). Contribution to the taxonomy and pathogenicity of fungicolous Verticillium species. I. Taxonomy. Netherlands Journal of Plant Pathology 88, 57-78.

Garcia Acha, I., Leal, J. A. \& Villaneuva, J. R. (1965). Lysis of uredospore germ tubes of rusts by species of Verticillium. Phytopatho$\log y 55,40-42$.

LIM, T. K. \& NiK, W. Z. (1983). Mycoparasitism of the coffee rust pathogen, Hemileia vastatrix, by Verticillium psalliotae in Malaysia. Pertanika 6, 23-25.

Mugnai, L., Bridge, P. D. \& Evans, H. C. (1989). A chemotaxonomic evaluation of the genus Beauveria. Mycological Research 92, 199-209.

Petch, T. (1925). Entomogenous fungi and their use in controlling insect pests. Bulletin of the Department of Agriculture, Ceylon no. 71. Colombo: Government Printer

PITT, J. I. (1980). The Genus Penicillium and its Teleomorphic States Eupenicillium and Talaromyces. London: Academic Press.

QUINLAN, R. J. (1988). Use of fungi to control insects in glasshouses. In Fungi in Biological Control Systems, pp. 19-36. Edited by M. N. Burge. Manchester: Manchester University Press.
Samson, R. A., Evans, H. C. \& Latge, J. P. (1988). Atlas of Entomopathogenic Fungi. Berlin: Springer-Verlag.

Van Der Schaaf, D. A., Malais, M. \& Ravensberg, W. J. (1990). The use of Verticillium lecanii against whitefly and thrips in glasshouse vegetables in the Netherlands. In Proceedings and Abstracts of the Sixth International Colloquium on Invertebrate Pathology and Microbial Control, p. 391. Adelaide, Australia: Society for Invertebrate Pathology.

SMITH, D. \& ONIONS, A. H. S. (1983). The Preservation and Maintenance of Living Fungi. Kew: Commonwealth Mycological Institute.

SNEATH, P. H. A. \& Sokal, R. R. (1973). Numerical Taxonomy. San Francisco: Freeman.

SPENCER, D. M. (1980). Parasitism of carnation rust (Uromyces dianthi) by Verticillium lecanii. Transactions of the British Mycological Society 74, 191-194.

Srivastava, A. K., Defago, G. \& Kern, H. (1985). Hyperparasitism of Puccinia horiana and other microcyclic rusts. Phytopathologische Zeitschrift 114, 73-78.

UMA, N. U. \& TAYLOR, G. S. (1987). Parasitism of leek rust urediniospores by four fungi. Transactions of the British Mycological Society 88, 335-340.

Woodbury, W., Spencer, A. K. \& Stahmann, M. A. (1971). An improved procedure using ferricyanide for detecting catalase isozymes. Analytical Biochemistry 44, 301-305. 